\title{
Tremendous Improvement of the Functional Capacity in Patient with Chronic Heart Failure after 12-Weeks of Exercise Training Program
}

\author{
Adam Staron', Jadwiga Wolszakiewicz', Mohammed Al Otaiby' ${ }^{1}$, Justyna Irla-Czyzycka², \\ Ibrahim Al Malki' ${ }^{1}$, Deemah Al-Khodairay ${ }^{1}$ \\ ${ }^{1}$ Cardiac Rehabilitation Unit, Prince Sultan Cardiac Center, Riyadh, KSA \\ ${ }^{2}$ Department of Cardiology, Cardiology Institute, Krakow, Poland \\ Email: astaron1973@wp.pl
}

How to cite this paper: Staron, A., Wolszakiewicz, J., Al Otaiby, M., Irla-Czyzycka, J., Al Malki, I. and Al-Khodairay, D. (2020) Tremendous Improvement of the Functional Capacity in Patient with Chronic Heart Failure after 12-Weeks of Exercise Training Program. Case Reports in Clinical Medicine, 9, 236-241.

https://doi.org/10.4236/crcm.2020.98033

Received: July 22, 2020

Accepted: August 21, 2020

Published: August 24, 2020

Copyright $\odot 2020$ by author(s) and Scientific Research Publishing Inc. This work is licensed under the Creative Commons Attribution International License (CC BY 4.0).

http://creativecommons.org/licenses/by/4.0/ (c) (i) Open Access

\begin{abstract}
Cardiac rehabilitation is recommended for patients with chronic heart failure (CHF) with preserved, or reduced left ventricular ejection fraction (LVEF). A cardiac rehabilitation program generally includes physical exercise, diet counseling, educational classes on lifestyle changes, and disease management as well as psychosocial support for patients and their families. Exercise training is a core component of the comprehensive cardiac rehabilitation program and is strongly recommended in combination with pharmacological treatment to patients with CHF, due to cardiorespiratory, metabolic, and autonomic cardiac response. Exercise-based cardiac rehabilitation affects positively functional capacity, exercise tolerance, and quality of life in CHF patients. The physical inactivity rate in Arabian Peninsula countries is extremely high, and exercise training of habitually physically inactive individuals should result in marked improvements of physical capacity. We present a case that demonstrates such a significant improvement in the physical capacity of a patient with CHF and a review of literature.
\end{abstract}

\section{Keywords}

Cardiac Rehabilitation, Chronic Heart Failure, Low-Intensity Interval Training, Functional Capacity

\section{Introduction}

Cardiac rehabilitation (CR), according to the World Health Organization guidelines, is defined as "the comprehensive and coordinated use of medical, social, educational and professional resources to accommodate patients to a new life- 
style and enable them to achieve the best performance" [1].

The cardiac rehabilitation program consists of physical exercise sessions, dietary counseling, educational classes on lifestyle changes, and psychosocial support to patients and their families.

Supervised exercise training remains a core component of the cardiac rehabilitation program, focusing on aerobic, resistance, neuromotor and flexibility components. Exercise training is strongly recommended for patients with chronic heart failure (CHF) at a class 1 evidence level [2].

The benefits of exercise-based cardiac rehabilitation (in terms of improved functional capacity, exercise tolerance and quality of life) have been widely recognized [3]. The exercise-based program has positive effects on reduction of the re-hospitalization rates and mortality [4]. Many studies of CHF patients have demonstrated improvements of $18 \%$ to $25 \%$ in peak oxygen uptake [5]. In a retrospective study by Martin, a cohort of 5400 patients with CHF was categorized into low, moderate and high cardiorespiratory fitness groups based on an initial exercise test. After 12-weeks of exercise training, an improvement of 1.4, 1.0, and 0.8 METs, respectively, were observed, with a greater improvement in the lowest fitness group [6]. The physical inactivity rate in the Kingdom of Saudi Arabia is extremely high, mainly due to weather and sociocultural barriers (frequent use of private drivers and housemaids, belief that exercise is not a social activity, lack of knowledge and/or motivation). Some-issues relate specifically to women as they are not expected to perform physical activity in public. Cardiac rehabilitation in the Kingdom of Saudi Arabia is still under development, therefore little is known about functional capacity improvement of habitually physically inactive Saudi patients. We observed a significant improvement of functional capacity (by 5.4 MET) in patient with CHF a severe impairment of the left ventricular systolic function (LVEF20\%).

\section{Case Report}

A 40-year-old male patient, an active smoker (10 cigarettes a day), diagnosed with dilated cardiomyopathy, arterial hypertension, dyslipidemia, and mild mitral regurgitation was referred to the Cardiac Rehabilitation Unit at Prince Sultan Cardiac Center, Riyadh. He complained of shortness of Breath at New York Heart Association (NYHA) class II on presentation in March 2019. The patient's medication regimen included Concor $10 \mathrm{mg}$ once a day, Aldactone $25 \mathrm{mg}$ once a day, Lasix $40 \mathrm{mg}$ once a day, Entresto $200 \mathrm{mg}$ twice a day, Aspirin $81 \mathrm{mg}$ once a day, and Lipitor $40 \mathrm{mg}$ once a day. The patient has been clinically stable on this treatment for the preceding few months.

During a physical examination, his heart rate, blood pressure, and oxygen saturation were within normal limits (blood pressure 109/60 $\mathrm{mmHg}$, heart rate 78 beats per minute, oxygen saturation $99 \%$ in room air).The lung examination showed no crepitations or rales. Chest $\mathrm{x}$-ray revealed cardiomegaly, no interstitial edema or pleural effusion. The heart assessment revealed a grade 2 systolic 
murmur over the apex area. The transthoracic echocardiography revealed a moderately dilated left ventricle and severe impairment of left ventricular systolic function (severe global hypokinesis, end-diastolic volume of $155 \mathrm{ml}$, left ventricular fractional shortening $12 \%$, ejection fraction of $20 \%$ ). There was also grade II diastolic dysfunction and mild mitral regurgitation. An initial graded stress test on a treadmill utilizing a modified Bruce protocol had been stopped due to significant shortness of breath after reaching 5.0 METs, which indicated low functional capacity, according to the American Association of Cardiovascular and Pulmonary Rehabilitation guidelines. The patient's exercise training program included low-intensity aerobic interval training and stretching exercises. Resistance training was implemented after 2 weeks of well-tolerated aerobic component. Aerobic training consisted of 5 minutes of warm-up exercise, followed by low-intensity interval training (LIIT) on treadmill, with a subsequent 5 -minute cool-down. The duration of the aerobic sessions was increased from 20 $\mathrm{min}$ in the first sessions to $40 \mathrm{~min}$ after 12 weeks. The duration of active phases as well as the recovery phases were 2 minutes, and after reaching 40 minutes of total training duration, training intensity was increased with an increase in speed for active phases of up to $3.5 \mathrm{~km} / \mathrm{h}$ (starting from $2 \mathrm{~km} / \mathrm{h}$ ). The training heart rate zone during active phases was set as $95-100 \mathrm{bpm}$. Training was monitored in terms of the patient's heart rate, electrocardiogram, and symptoms. Blood pressure was monitored before and after training. Resistance training was initiated after 2 weeks and included an initial work-to-rest ratio of 1:2, i.e. 30 - 60 seconds of exercise with a subsequent 1 -2-minute rest. Full resistance training progression was reached after 4 weeks. Patient before presentation to Cardiac Rehabilitation Unit underwent already respiratory (inspiratory muscle) training 3 times as week 20 min for 8 weeks. All exercise training components have been demonstrated in Table 1 Following the 12-week program, both physical capacity and echocardiographic parameters of the left ventricular systolic function significantly improved. The control echocardiogram revealed end-diastolic volume of $147 \mathrm{ml}$, left ventricular fractional shortening of $15 \%$, and left ventricular ejection fraction of $27 \%$. Control graded stress test on treadmill utilizing the modified Bruce protocol had been terminated due to significant shortness of breath after reaching 10.4 METs. Both exercise tests were terminated at similar heart rate levels (125 bpm and $128 \mathrm{bpm}$, respectively).

\section{Discussion/Review of Literature}

\subsection{Role of Exercise Training in Patients with CHF}

The loss of physical function in a patient with heart failure is well documented and contributes to difficulties in performing daily living tasks. The impairment of functional capacity in CHF individuals is a result of impaired cardiac output response to exertion, impaired vasodilation, and increased systemic vascular resistance [7]. Skeletal muscle dysfunction (reductions in muscle mass, strength, and endurance) also contributes to the increased fatigue and exercise intolerance 
Table 1. Training components.

\begin{tabular}{|c|c|c|c|c|}
\hline Training mode & Duration & Frequency & Intensity & Type \\
\hline $\begin{array}{c}\text { Aerobic } \\
\text { (with warm-up } \\
\text { and cool-down) }\end{array}$ & $\begin{array}{l}\text { Initial: } 20 \text { minutes } \\
\text { Final: } 40 \text { minutes }\end{array}$ & Twice a week & $\begin{array}{c}\text { Initial: } 40 \%-50 \% \text { of } \\
\text { heart rate reserve } \\
\text { Final: } 50 \%-60 \% \text { of } \\
\text { heart rate reserve }\end{array}$ & $\begin{array}{l}\text { Walking } \\
\text { On } \\
\text { teadmill }\end{array}$ \\
\hline $\begin{array}{c}\text { Resistance } \\
\text { (after stretching) }\end{array}$ & $\begin{array}{l}\text { Initial: } 10 \text { minutes } \\
\text { Final: } 20 \text { minutes }\end{array}$ & Twice a week & $\begin{array}{l}30 \%-40 \% \text { of } \\
\text { 1-repetition } \\
\text { maximum } 1 \text { set } \\
8 \text { - } 12 \text { repetitions } \\
8 \text { muscle groups }\end{array}$ & $\begin{array}{l}\text { Using dumbbells } \\
\text { and elastic bands }\end{array}$ \\
\hline $\begin{array}{c}\text { Stretching } \\
\text { (after aerobic and } \\
\text { resistance part) }\end{array}$ & $\begin{array}{c}5 \text { minutes in total } \\
2 \times 30-60 \mathrm{sec} \\
\text { stretches }\end{array}$ & Twice a week & $\begin{array}{l}\text { Stretch to a point of } \\
\text { feeling tightness or } \\
\text { slight discomfort }\end{array}$ & Static flexibility \\
\hline
\end{tabular}

in this population. Exercise training is strongly recommended for patients with CHF; and as the non pharmacological intervention has become widely accepted, with both aerobic and resistance exercise being shown to be efficacious in CHF patients. Exercise training in patients with CHF is associated with reduction of sympathetic activity (reduced levels of plasma norepinephrine and muscle sympathetic nerve activity) and an increase in parasympathetic tone (increase in heart rate variability and baroreflex sensitivity).

Besides, regular exercise improves endothelium-dependent vasodilatation and has anti-inflammatory effect [8].

Beneficial effects of exercise training include an increase in the maximal oxygen uptake by $(2.1 \mathrm{ml} / \mathrm{kg} / \mathrm{min})$, prolonged exercise duration (by $2.3 \mathrm{~min}$ ), increase in peak work (by 15 Watts) or walking distance in a 6 min walk test [9].

\subsection{Exercise Prescription for Patients with $\mathrm{CHF}$}

An appropriate exercise prescription in patients with heart failure is complex and requires knowledge about underlying causes, recent pharmacotherapy, and the current status of the patient's functional capacity. The initial exercise intensity should be optimally based on the cardiopulmonary exercise test (CPET) with an initial training intensity set at $40 \%-50 \%$ of peak oxygen uptake, which can eventually be progressed to $70 \%-80 \%$. If CPET is unavailable, an intensity of $40 \%-70 \%$ of the heart rate reserve and Borg scale $10-14$ are recommended. Ideally, an initial work-to-recovery time ratio of 1:2 or 1:1 is recommended. Active phases can be as short as $10-30 \mathrm{sec}$ initially, and recovery phases should be rather passive (0 Watts for cycle ergometer, $1-2 \mathrm{~km} / \mathrm{h}$ speed for treadmill).

Training intensity can be increased in the total time and/or a change in the work-to-recovery time (i.e., prolonged duration of active segments and/or by shortening the recovery time).

\subsection{Review of Literature}

As mentioned earlier, studies in CHF patients have demonstrated improvements 
of $18 \%$ to $25 \%$ in peak oxygen consumption and $18 \%$ to $34 \%$ in peak exercise duration with improvement in symptoms, functional class, and quality of life [10]. It is related to increased cardiac output (by prolonged ventricular filling and higher stroke volume) and oxygen extraction by working the muscles. Exercise training in patients with $\mathrm{CHF}$ and reduced left ventricular ejection fraction $(<35 \%)$ was associated with a reduced incidence $(11 \%)$ of the combined all-cause mortality or hospitalization [11]. As discussed earlier, the lower the initial cardiorespiratory fitness the greater the final improvement. It has been documented that aerobic exercise can lead to improvement of LVEF by $2.5 \%$ [12].

\subsection{Training Results and Discussion}

In the case of our patient, aerobic training had been interval in nature, which is consistent with the finding that the interval training group showed higher oxygen consumption, more pronounced reverse left ventricular remodeling, or an increase in $\mathrm{EF}$ compared to continuous moderate-intensity exercise training in CHF patients [13]. We implemented resistance training after 2 weeks of well-tolerated cardiorespiratory (aerobic) component. As mentioned earlier, after 12 weeks of exercise training, the functional capacity of the patient tremendously increased from 5.0 to 10.4 METs. There was also a significant improvement of left ventricular systolic function (LVEF improved by 7\%). The physical inactivity rate in Arabian Peninsula countries is extremely high, with the highest prevalence in the Kingdom of Saudi Arabia, with, specifically, $61 \%$ of males and $73 \%$ of females being physically inactive [14] [15]. Two of the most reported barriers to physical activity are weather and sociocultural barriers.

Therefore exercise training of habitually physically inactive individuals may result in such significant improvement of physical capacity, and in prominent reduction of cardiovascular mortality. Obviously the development of cardiac rehabilitation services throughout the Kingdom of Saudi Arabia could be a real milestone in the treatment of patients with cardiovascular diseases. Although case reports are lowest level of evidence, we observe at our center on regular basis such a significant improvement of physical capacity in patients with CHF.

\section{Conflicts of Interest}

The authors declare no conflicts of interest regarding the publication of this paper.

\section{References}

[1] American Association of Cardiovascular and Pulmonary Rehabilitation (2020) Guidelines for Cardiac Rehabilitation and Secondary Prevention Programs. 6th Edition, Human Kinetics Publishers, Champaign.

[2] Ambrosetti, M., Abreu, A., Corra, U., Davos, C.H., Hansen, D., Frederix, I., et al. (2020) Secondary Prevention through Comprehensive Cardiovascular Rehabilitation: From Knowledge to Implementation. 2020 Update. A Position Paper from the Secondary Prevention and Rehabilitation Section of the European Association of 
Preventive Cardiology. European Journal of Preventive Cardiology. https://doi.org/10.1177/2047487320913379

[3] Downing, J., Gary, J. and Balady, G.J. (2011) The Role of Exercise Training in Heart Failure. JACC, 58, 561-569. https://doi.org/10.1016/j.jacc.2011.04.020

[4] Keteyian, S.J., Leifer, E.S., Houston-Miller, N., et al. (2012) Relation between Volume of Exercise and Clinical Outcomes in Patients with Heart Failure. JACC, 60, 1899-1905. https://doi.org/10.1016/j.jacc.2012.08.958

[5] Flynn, K.E., Pina, I.L., Whellan, D.J., Lin, L., Blumenthal, J.A., Ellis, S.J., et al. (2009) Effects of Exercise Training on Health Status in Patients with Chronic Heart Failure: HF-ACTION Randomized Controlled Trial. JAMA, 301, 1451-1459. https://doi.org/10.1001/jama.2009.457

[6] Martin, B.J., Arena, R., Haykowsky, M., et al. (2013) Cardiovascular Fitness and Mortality after Contemporary Cardiac Rehabilitation. Mayo Clinic Proceedings, 88, 455-463. https://doi.org/10.1016/j.mayocp.2013.02.013

[7] Ribeiro, J.P., Chiappa, G.R., Neder, J.A. and Frankenstein, L. (2009) Respiratory Muscle Function and Exercise Intolerance in Heart Failure. Current Heart Failure Reports, 6, 95-101. https://doi.org/10.1007/s11897-009-0015-7

[8] Gademan, M.G., Swenne, C.A., Verwey, H.F., van der Laarse, A., Maan, A.C., van de Vooren, H., van Pelt, J., van Exel, H.J., Lucas, C.M., Cleuren, G.V., Somer, S., Schalij, M.J. and van der Wall, E.E. (2007) Effect of Exercise Training on Autonomic Derangement and Neurohumoral Activation in Chronic Heart Failure. Journal of Cardiac Failure, 13, 294-303. https://doi.org/10.1016/j.cardfail.2006.12.006

[9] Belardinelli, R., Georgiou, D., Cianci, G. and Purcaro, A. (1999) Randomized, Controlled Trial of Long-Term Moderate Exercise Training in Chronic Heart Failure: Effects on Functional Capacity, Quality of Life, and Clinical Outcome. Circulation, 99, 1173-1182. https://doi.org/10.1161/01.CIR.99.9.1173

[10] Flynn, K.E., Pina, I.L., Whellan, D.J., Lin, L., Blumenthal, J.A., Ellis, S.J., et al. (2009) Effects of Exercise Training on Health Status in Patients with Chronic Heart Failure: HFACTION Randomized Controlled Trial. JAMA, 301, 1451-1459. https://doi.org/10.1001/jama.2009.457

[11] O'Connor, C.M., Whellan, D.J., Lee, K.L., et al. (2009) Efficacy and Safety of Exercise Training in Patients with Chronic Heart Failure: HF-ACTION Randomized Controlled Trial. JAMA, 301, 1439-1450. https://doi.org/10.1001/jama.2009.454

[12] Haykowsky, M., Liang, Y., Pechter, D., Jones, L., McAlister, F. and Clark, A. (2007) A Meta-Analysis of the Effect of Exercise Training on Left Ventricular Remodeling in Heart Failure Patients: The Benefit Depends on the Type of Training Performed. Journal of the American College of Cardiology, 49, 2329-2336. https://doi.org/10.1016/j.jacc.2007.02.055

[13] Wisloff, U., Stoylen, A., Loennechen, J.P., Bruvold, M., Rognmo, O., Haram, P.M., et al. (2007) Superior Cardiovascular Effect of Aerobic Interval Training versus Moderate Continuous Training in Heart Failure Patients: A Randomized Study. Circulation, 115, 3086-3094. https://doi.org/10.1161/CIRCULATIONAHA.106.675041

[14] Mabry, R., Reeves, Eaking, E. and Owen, N. (2009) Evidence of Physical Activity Participation among Men and Women in the Countries of the Gulf Cooperation Council: A Review. Obesity Reviews, 11, 457-464. https://doi.org/10.1111/j.1467-789X.2009.00655.x

[15] Kahan, D. (2015) Adult Physical Inactivity Prevalence in the Muslim World: Analysis of 38 Countries. Preventive Medicine Reports, 2, 71-75. https://doi.org/10.1016/j.pmedr.2014.12.007 